\title{
VALIDEZ DE Potamides munieri LARRAZET, 1894 (GASTERÓPODO DEL MIOCENO DE BURGOS)
}

\author{
Sebastián CALZADA', Jaime de PORTA² y Jaime TRUYOLS \\ ' Museo Geológico del Seminario de Barcelona. Diputación 231. 08007 Barcelona. \\ 'Departament de Estratigrafia i Paleontologia. Facultat de Geologia. Grup de Paleobiologia del Neògen \\ Mediterrani 1999. SGR 348. Universitat de Barcelona. \\ ${ }^{3}$ Departamento de Geología. Universidad de Oviedo.
}

Calzada, S., Porta, J. de y Truyols, J. 2001. Validez de Potamides munieri Larrazet, 1894 (gasterópodo del Mioceno de Burgos). [Validity of Potamides munieri Larrazet, 1894 (gastropod from the Miocene of Burgos, Spain)]. Revista Española de Paleontología, 16(1), 161-165. ISSN 0213-6937.

\begin{abstract}
In 1894 Potamides gaudryi and Potamides munieri were described by Larrazet as new species. They were collected from Miocene beds of Castrillo del Val (Burgos Province), eastwards Duero river depression. Royo Gómez (1925) regarded these species as synonyms of Potamides tricinctus. P. tricinctus, type species of the subgenus Ptychopotamides, is characterized by the presence of a columelar fold. However, this columelar fold does not exist in the Potamides species described by Larrazet. The existence of transitional forms between $P$. gandry and $P$. munieri leads us to consider both species as belonging to a single one, Potamides munieri. The grounds for this systematic revision are discussed in this paper.
\end{abstract}

Keywords: Mollusca, Gastropoda, Ceritoidea, revision, Miocene, Duero Basin, Spain.

\section{RESUMEN}

En 1894 Larrazet describió como nuevas especies Potamides gaudryi y Potamides munieri. Ambas procedían del Mioceno de Castrillo del Val (Provincia de Burgos), depresión del Duero. Royo Gómez (1925) las consideró como sinónimas de Potamides tricinctus. P. tricinctus es la especie tipo del subgénero Ptychopotamides, caracterizado por tener un pliegue columelar. Sin embargo, las especies de Potamides descritas por Larrazet no poseen pliegue columelar. La existencia de formas de transición entre $P$. gaudryi y $P$. munieri nos lleva a reunir ambas en una sola especie, $P$. munieri. Todo ello se expresa al modo de una revisión sistemática.

Palabras clave: Mollusca, Gastropoda, Ceritoidea, revisión, Mioceno, Cuenca del Duero, España.

\section{INTRODUCCIÓN}

En 1894, Larrazet estudió el rico yacimiento fosilífero de Castrillo del Val (Provincia de Burgos) situado en el extremo nordeste de la Depresión del Duero. Entre otras especies describió como nuevas Potamides gaudryi y Potamides munieri. Posteriormente, Royo Gómez (1925), tras estudiar tanto los materiales de Larrazet (1894) como otros de diversos Museos, concluyó que esos Potamides eran sinónimos de Potamides tricinctus (Brocchi). Autores posteriores han estado de acuerdo con la atribución de Royo Gómez (1925), pero en 1989 Lozouet (in Anadón, 1989) los llama Potamides cf. disjunctus, gr. lamarcki. Esta disparidad de criterios obliga a replantear o revisar la cuestión a la que también invita la magnífica conservación de los ejemplares y por otra parte el cuidadoso e inteligente estudio de Larrazet (1894). Previamente se reproduce la historia de esas especies.

\section{HISTORIA}

Larrazet (1894) es el primer autor que se ocupó de este yacimiento, del que erigió dos especies nuevas de Potamides: Potamides gaudryi y Potamides munieri. En esta última distinguió cuatro variedades, definidas por el mayor o menor relieve de la ornamentación. Se destaca que Larrazet no dio valor específico a este último carácter.

Royo Gómez (1925) tras visitar múltiples museos de Francia, Suiza y Alemania, comparando los ejemplares de Castrillo con los de sus colecciones, concluye que “j”ai 


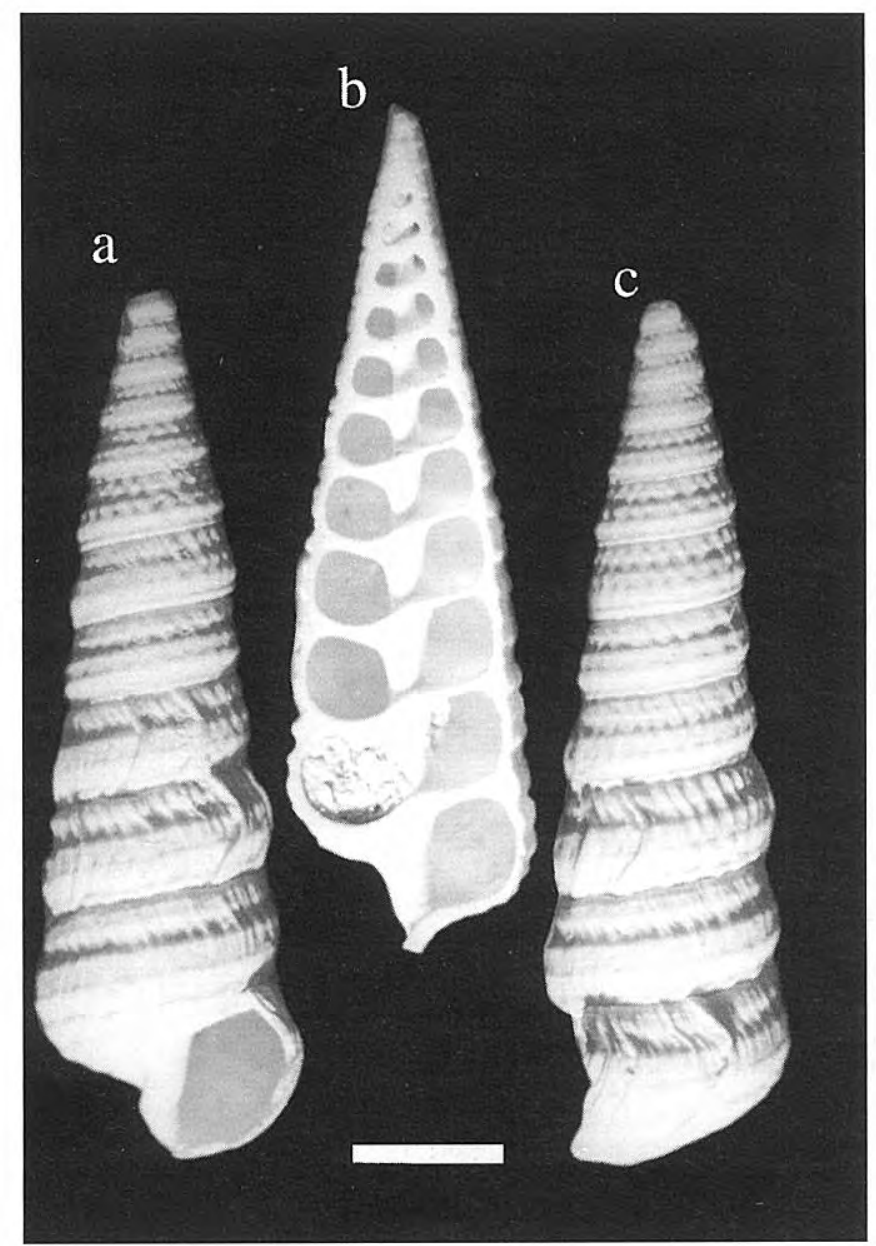

Figura 1. Potamides munieri Larrazet, 1894. Astaraciense de Castrillo del Val (Provincia de Burgos). a: Vista adapertural. b: Sección mostrando la ausencia de pliegue columelar. c: Vista lateral. Escala: $1 \mathrm{~cm}$. (Foto Farrés Malian), a y c: ejemplar número 66894.21. b: ejemplar número 66894.2. Todos ellos depositados en el Museo geológico del Seminario de Barcelona.

Potamides munieri Larrazet, 1894. Astaracian of Castrillo del Val (Burgos Province). a: adapertural view. $\boldsymbol{b}$ : cut showing the absence of $\boldsymbol{a}$ columelar fold. c: lateral view. Scale: $1 \mathrm{~cm}$. (Photo Farrés Malian). a and c: specimen 66894.21. b: specimen 66894.2. All are housed in the Geological Museum of the Seminary of Barcelona.

pu constater que les Potamides sont certainement Potamides tricinctus $\mathrm{Br}$. avec passage à Potamides papaveraceum Bast. et même à $P$. basteroti var. africana Tourn." Royo Gómez parece valorar exclusivamente la ornamentación, ya que no menciona otros caracteres. Un trabajo posterior de Royo Gómez (1926) no aporta nuevos datos a esa asimilación.

Truyols y de Porta (1982) publican un trabajo recapitulativo y con incursiones tafonómicas y ecológicas. Expresamente advierten que no pretenden una revisión de la fauna. Aceptan la opinión de Royo Gómez en lo tocante a los Potamides.
Ibáñez (1984) publica un estudio divulgativo sobre el Mioceno de Castrillo del Val, en el que reitera las opiniones de Royo Gómez (op. cit.) y de la Memoria de la Hoja 20, Burgos (escala 1:200.000). Figura dos Potamides sp., pero entre los fósiles recogidos cita Potamides tricinctus.

Anadón (1989) resume todas las aportaciones a la fauna de Castrillo del Val y recuerda la atribución de Royo Gómez (op. cit.) a Potamides tricinctus. Sin embargo, en la lámina 1 consta la determinación de Lozouet según la cual los Potamides son Potamides cf. disjunctus (gr. lamarcki). En el texto del artículo este dato sólo consta en la leyenda castellana de la tabla 1: "Se ha mantenido la denominación taxonómica empleada en las citas bibliográficas, excepto para (1) Potamides gr. lamarcki, según Lozouet in litt”. Es decir, Anadón sugiere una modificación a la atribución a P. tricinctus.

García de Domingo (1997), redactor de la Memoria de la Hoja 238 (Villagonzalo-Pedernales), resume los datos de Truyols y de Porta (1982), repitiendo la presencia de Potamides tricinctus.

\section{SISTEMÁTICA}

Familia Potamididae H. y A. Adams, 1854

Subfamilia Potamidinae H. y A. Adams, 1854

Género Potamides Brongniart, 1810

Subgénero Potamides (Potamides) Brongniart, 1810

1906 Potamides Brongniart; Cossmnann, 103.

1938 Potamides Brongniart; Wenz, 736.

1986 Potamides Brongniart; Lozouet, 166 y passim.

1998 Potamides Brongniart; Kowalke, 28.

Especie tipo: Potamides lamarcki Brongniart, 1810.

\section{Sobre el género y el subgénero}

Según Wenz (1938) el género Potamides se subdivide en seis subgéneros. De éstos, sólo dos tienen representantes miocénicos. Estos dos subgéneros son Potamides y Ptychopotamides. Potamides no tiene pliegue en su columela. Por el contrario, Ptychopotamides muestra uno en la parte media de la columela. Glibert (1962) recalca que el mejor carácter distintivo de Ptychopotamides es "la présence d'un pli spiral interne s'enroulant sur toute la hauteur de la columelle; il est souhaitable de verifier ce caractère par une coupe longitudinal du test". Hemos pulido varios ejemplares de Castrillo del Val para comprobar la posible existencia de ese pliegue columelar. El resultado ha sido negativo.

Larrazet (1894, p. 377) observa que: "La columnelle n'est pas plissée; cependant, elle présente des traces presque imperceptibles de plis en certains points de sa longueur." Esta última afirmación se considera que tributa más a la escrupulosidad en la observación que no a una realidad objetiva de pliegues en la columela. Hemos observado esas trazas en algunos ejemplares, pero que no representan un verdadero pliegue. Por todo lo cual, concluimos que los Potamides de Castrillo, al no 
poseer pliegue columelar, no pueden asimilarse a Ptychopotamides. Sin embargo, repetimos que la torsión de la columela puede sugerir la presencia de un pliegue. Véase, por ejemplo, la figura $2 \mathrm{G}$ de Lozouet (1986, p. 171). Esto explicaría la opinión de Royo Gómez.

De la multitud de juicios que expresa Lozouet (1986), sólo destacamos dos. Primero: Ptychopotamides como subgénero válido de Potamides, "Ptychopotamides (Sacco, 1885) qui groupe les espèces à trois rangées de granulations et à fort pli columellaire (type tricinctus Brocchi du Pliocène italien)". (Lozouet 1986, p. 167). Segundo: El escaso valor de la ornamentación en la clasificación. (Lozouet 1986, p. 168). Otras cuestiones están fuera del objeto de esta nota, aunque se echa en falta la referencia a los Potamides de Larrazet (op. cit.) en la enumeración de especies.

\section{Potamides (Potamides) munieri Larrazet, 1894} Fig. 1.

1894 Potamides gaudryi Larrazet, 376, pl. 13, figs. 1 y 1 a. 1894 Potamides munieri Larrazet, 376, pl. 13, figs. 3 a 23. 1897 Potamides gaudryi Larrazet, 139, lám. 30, figs. 1 y 1a. 1897 Potamides munieri Larrazet, 139, lám. 30, figs. 3 a 10 y lám. 31, figs. 12 a 23.

1926 Potamides tricinctum Brocchi; Royo Gómez, 36, lám. 7.

1982 Potamides (Ptychopotamides) tricinctum Brocchi; Truyols y de Porta, 670, fig. 3.

1984 Potamides sp.; Ibáñez, 240, (figura superior).

1989 Potamides cf. disjunctus (gr. lamarcki); Lozouet in Anadón, lám. 1, fig. d.

\section{Datos taxonómicos}

Larrazet (op. cit.) describió y figuró la especie muy bien. Suponemos que el holotipo se conserva en la colección Lazarret depositada en La Sorbona (fide Royo Gómez, 1925). Razón: Royo Gómez estudió la colección de Larracet en París; dice que visitó La Sorbona y la Escuela de Minas. Dado que en la recopilación de Prieur (1980), que incluye los fondos de la Escuela de Minas, no consta esa colección, deducimos que debe hallarse en La Sorbona. A pesar de nuestras pesquisas, no se ha podido conseguir el número de registro ni tampoco la certeza de su existencia.

Atendiendo a que Potamides munieri tiene entre sus variedades ejemplares con sólo dos cordones, carácter que define a Potamides gaudryi, se considera que Potamides gaudryi y Potamides munieri forman un solo conjunto específico, que se denomina Potamides munieri. Recordemos que el mismo Larrazet (op. cit.) advierte que existen formas de transición entre las dos especies y sus variedades. Por todo ello, parece oportuno ver en todo ese conjunto una sola especie, ciertamente muy polimorfa.

\section{Edad}

Aquitaniense según Larrazet (op. cit.). "Sarmatien supérieur et le Pontien inférieur" según Royo Gómez (1925, p. 84). "Vindoboniense superior" según Truyols y de Porta (1982). "Mioceno superior" según Lozouet in Anadón (1989). Para García de Domingo (1997), redactor de la Memoria de la hoja
238, los yacimientos de Castrillo del Val pertenecen al Astaraciense superior, sin excluir el Vallesiense basal. Nos parece adecuada esta atribución.

\section{Localidad tipo}

Castrillo del Val (Prov. de Burgos). Hoja 238, Villagonzalo de Pedernales, también escrito Villagonzalo-Pedernales. Coordenadas: $3^{\circ} 35^{\prime} 04^{\prime}$ ' y $42^{\circ} 19^{\prime} 06^{\prime}$ '. Los campos inmediatos al pueblo contienen abundantes ejemplares, sobre todo en el camino al Cementerio. Para los pormenores sedimentológicos nos remitimos a Sánchez Benavides et al. (1985). Para los estratigráficos, a la Memoria de la Hoja 238 (García de Domingo, 1997).

\section{Diagnosis}

Especie del género Potamides, que en general tiene 3 cordones espirales ( 2 a 4 extremos), con tubérculos o lisos, y sin pliegue en la columela.

\section{Descripción original}

Potamides gaudryi: "Coquille allongée, turriculée, conique, pointue à la partie inférieure, composée de nombreux tours assez aplatis, chacun de ces derniers est orné de deux larges côtes tuberculées. Les tubercules de la première côte sont nombreux, gros, plus ou moins arrondis et atteignent le bord inférieur de chaque tour; ceux de la seconde côte sont aussi nombreux, mais ils sont sensiblement moins gros que les précédents, surtout à la partie moyenne et supérieure de la coquille et n'atteignent pas tout à fait le bord supérieur du tour. Le sillon qui existe entre ces deux ranges de tubercules est à peu près aussi large que ces derniers et est traversé par des larges lignes relativement peu proéminentes réunissant deux à deux les tubercules des deux rangs. Le péristome est ovale, presque arrondi; le dernier tour est déprimé à sa partie supérieure, où il est orné de quatre côtes longitudinales au-dessus de la seconde côte tuberculée."

Potamides munieri: "Coquille allongée, turriculée, conique, et composée d'un assez grand nombre de tours. Ces tours sont généralment plus ou moins plans; mais parfois ils deviennent convexes ou présentent une ou deux carènes plus ou moins nettes; l'angle spiral, généralement faible, offre cependant quelques remarquables variations. Les caractères du péristome et du dernier tour sont les mêmes que ceux que j'ai constatés pour l'espèce précédente. La columnelle n'est pas plissée; cependant, elle présente des traces presque imperceptibles de plis en certains points de sa longueur".

"Chaque tour est orné de trois côtes complètement tuberculées. Assez souvent, les tubercules les plus faibles sont ceux du second rang, puis viennent ceux du troisième et enfin ceux de premier; mais cette différence n'existe pas toujours. Les tours peuvent avoir des ornaments transversaux: ce sont des lignes d'acroissement ou de petites côtes qui réunissent deux à deux les tubercules correspondants des trois côtes longitudinales". Este texto está copiado de parte de las descripciones de Potamides munieri n. sp. y de Potamides munieri s. str.

\section{Descripción complementaria}

La descripción original de Larrazet (op. cit.) es válida y da cabal cuenta de la especie. Aquí sólo se completa con algunas 
observaciones, tanto cualitativas como cuantitativas, tomadas del estudio de más de un centenar de topotipos, depositados en el Museo Geológico del Seminario de Barcelona con el número 66894.

Protoconcha raramente conservada. Un botón heterostrófico y sumergido seguido de una vuelta completa.

Las líneas de crecimiento son muy visibles, acentuadas a veces por seudovarices lineares y son claramente opistocirtas.

Ordinariamente conservan el color anaranjado oscuro, pero existen ejemplares de color prácticamente blanco.

La abertura, que raras veces se ha conservado intacta, es ovalada con el peristoma encallecido en su parte columelar y parietal. De acuerdo con la disposición opistocirta de las líneas de crecimiento, el labio externo es cóncavo, dilatándose en su parte más abapical y se retrae contorneando en su parte más adaxial el sifón. En su contacto con la columela dibuja un ángulo de unos $90^{\circ}$. La columela no es recta, y está algo más torcida en su parte adapical delimitando el cortísimo canal sifonal.

La base es algo convexa y está recubierta por ligeros cordones espirales, granulosos, más acentuados los más periféricos, y por líneas axiales de crecimiento, que a veces adquieren el papel dominante.

Datos numéricos. Como simple orientación y sin pretensiones estadísticas, se dan las medidas de un conjunto de 25 ejemplares de longitud superior a $19 \mathrm{~mm}$, valor elegido arbitrariamente, como límite inferior de los individuos adultos ya que el $80 \%$ de los ejemplares vistos (más de un centenar) supera esa longitud.

Valor medio de la longitud o altura, (límite acotado $19 \mathrm{~mm}$ ) $=\mathrm{H}=27,7$ (recorrido: 19 a 40).

Valor medio de la anchura $=\mathrm{W}=8,4$ (recorrido: 6 a 11).

Valor medio de la longitud de la última vuelta $=\mathrm{u}=8,8$ (recorrido: 6,5 a 12).

Valor medio de la relación $\mathrm{W} / \mathrm{H}=$ anchura/altura $=0,31$ (recorrido: 0,28-0,36).

Valor medio de la relación $\mathrm{u} / \mathrm{H}=$ altura de la última vuelta/altura total $=0,29$ (recorrido: $0,25-0,38$ ).

Valor medio del número de gránulos por cordón en la última vuelta: 25 (recorrido: 23 a 26).

Valor medio de la relación altura vuelta/anchura vuelta: 0,53 (recorrido: 0,49 a 0,57).

\section{Variabilidad}

Larrazet (op. cit.) insiste en la gran variabilidad de Potamides. Esta variabilidad se halla tanto entre los diversos ejemplares de una población como en las diferentes vueltas de un mismo individuo. Los aspectos más importantes son:

- el número de cordones espirales, entre 2 y 4, dominando el número de 3.

- el tamaño de los tubérculos en los cordones. Aquéllos pueden casi desaparecer y en este caso los cordones se convierten en carenas débilmente granulosas.

- el perfil de vuelta, que puede ser plano, convexo o de doble plano inclinado.

- el valor del ángulo espiral, que puede variar unos $10^{\circ}$ o más.

- la forma general, claramente aciculada o pupoide.

- la presencia de varices lineares, y su número y posición.

Esta varibilidad puede explicarse quizá, por adaptaciones ecológicas.

\section{Comparación con otras especies}

Por no tener un pliegue columelar, Potamides munieri no puede incluirse en el subgénero Ptychopotamides, y así se distingue fácilmente de Potamides (Ptychopotamides) tricinctus, que es la especie tipo del subgénero. Además, esa especie fue descrita del Plioceno y parece ser propia de esa serie. La "Crete seniensi", de la descripción original de Brocchi (1814) completada por Rossi Ronchetti (1955), corresponde al Plioceno. Wenz (1938) figura un ejemplar proveniente del Plioceno superior. Glibert (1962, p. 160) cita Potamides tricinctus en el Plasenciense de Italia y en el Scaldisiense e Iceniense de Gran Bretaña.

Potamides (Ptychopotamides) papaveraceus (Basterot, 1825) muestra también un pliegue columelar y así se distingue de los ejemplares de Castrillo del Val. Aunque su ornamentación es casi igual: tres cordones con unos 25 gránulos cada uno.

Nota

Todas las obras consultadas expresan la referencia a Larrazet de dos formas. Una sin la inicial del nombre y la otra con la inicial de M. Esto último es una mala lectura de la manera como se expresaba entonces la autoría. La M. es la abreviatura de Monsieur. En las dedicatorias autógrafas de Larrazet a Almera aparece antes del apellido una A. Esta "A" consta también en los correspondientes índices del Bulletin de la Société Géologique de France. No sabemos cuál era el onomástico de Larrazet. Sólo sabemos que empezaba por A.

\section{CONCLUSIÓN}

Los Potamides de Castrillo del Val (Burgos) pertenecen a una sola especie: Potamides munieri Larrazet, 1894. Por carecer de pliegue columelar, no se acepta que sea un sinónimo de Potamides (Ptychopotamides) tricinctus donde sí existe ese pliegue.

\section{AGRADECIMIENTOS}

Al Dr. Iginio Dieni de Padova por datos sobre P. tricinctus. A D. Francesc Farrés Malian por sus fotos. Al Dr. Luis Sebastián Alafont por su ayuda en la consulta bibliográfica.

\section{BIBLIOGRAFÍA}

Anadón, P. 1989. Los lagos salinos interiores (atalásicos) con faunas de afinidad marina del Cenozoico de la Península Ibérica. Acta Geologica Hispanica, 24, 83102.

Basterot, B. de. 1825. Description géologique du bassin tertiaire du Sud-Ouest de la France. Mémoires de la Société d'Histoire naturelle de Paris, 2, 1-100, 7 pls.

Brocchi, G. B. 1814. Conchiologia fossile subappennina con observazioni geologichi sugli Appenini e sul suolo adiacente. Stamperia Reale, Milán, 2 vols., 677 pp., 16 tav 
Brongniart, A. 1810. Sur les terrains qui paroissent avoir formés sous l'eau douce. Annales du Muséum de l'Histoire naturelle de Paris, 15, 357-405.

Cossmann, M. 1906. Essais de Paléoconchologie comparée. Septième livraison, París, 262 pp., 14 pls.

García de Domingo, A. 1997. Memoria de la Hoja 238, Villagonzalo-Pedernales del Mapa Geológico de España. Escala 1:50.000. Instituto Tecnológico Geominero de España, Madrid, 109 pp.

Glibert, M. 1962. Les Mesogasteropoda fossiles du Cénozoïque étranger des collections de l'Institut royal des Sciences Naturelles de Belgique. Mémoires de l'Institut royal des Sciences Naturelles de Belgique, Deuxième série, 69, 1-305.

Ibáñez, R. 1984. Estudi i situació del Miocé de Castrillo del Val (Burgos). Mineralogistes de Catalunya, 2 (9), $237-$ 241.

Instituto Geológico y Minero de España. 1970. Hoja 20, Burgos. Mapa Geológico de España, E. 1:200.000. Síntesis de la cartografía existente. Instituto Geológico y Minero de España, Madrid, 33 pp.

Kowalke, T. 1998. Bewertung protoconchmorphologischer Daten basaler Caenosgastropoda (Cerithiimorpha und Littorinimorpha) hinsichtlich ihrer Systematik und Evolution von der Kreide bis Rezent. Berliner Geowissensschaftliche Abhandlungen, Reihe E, 27, 1121.

Larrazet, A. 1894. Notes stratigraphiques et paléontologiques sur la province de Burgos. Bulletin de la Société Géologique de France, Paris, 3 série, 22, 366384, pl. 13. Traducción española (Marcial de Olavarría) en el Boletín de la Comisión del Mapa geológico de España, 22; serie 2a , tomo 2: 121-143, láms. 30 y 31. Madrid, 1897.
Lozouet, P. 1986. Redéfinition des genres Potamides et Pirenella (Gastropoda, Prosobranchia) à partir des espèces actuelles et fossiles: Implications phylétiques et biogéographiques. Annales de Paléontologie, 72, 163210.

Prieur, A. 1980. Catalogue des collections paléontologiques françaises. Office National de Gestion des Collections Paléontologiques françaises. Département des Sciences de la Terre. Université Claude Bernard, Lyon I. 308 pp.

Rossi Ronchetti, C. 1955. I tipi della "Conchiologia fossile subappennina" di G. Brocchi. II, Gastropodi, Scafopodi. Rivista Italiana di Paleontologia e Stratigrafia, Memoria 5, parte II, 91-343, + índices.

Royo Gómez, J. 1925. Notes sur la géologie de la Péninsule Ibérique. Bulletin de la Société Géologique de France, 4 série, 25, 83-88.

Royo Gómez, J. 1926. Excursión A-6. Terciario continental de Burgos. XIV Congreso Geológico Internacional. Instituto Geológico de España, Madrid, 69 pp.

Sacco, F. 1895. I molluschi dei terreni terziari del Piemonte e della Liguria. 17, Carlo Clausen. Turín, 1-52.

Sánchez Benavides, F.J., Alonso, G. y Dabrio, C.J. 1988. Sedimentología de los depósitos lacustres neógenos de Castrillo del Val (Burgos). Acta Geologica Salmaticensia, 25, 87-108.

Truyols, J. y Porta, J. de. 1982. Observaciones sobre los niveles fosilíferos del Mioceno de Castrillo del Val (Burgos). Temas Geológicos Mineros. IGME, VI, parte $2^{a}$. Primera reunión sobre la Geología de la Cuenca del Duero, Salamanca 1979. Madrid, 663-676.

Wenz, W. 1938. Gastropoda. Teil I. Allgemeiner Teil und Prosobranchia I. In: Handbuch der Paläozoologie (Ed. O.H. Schindewolf). Band 6. Gebrüder Borntraeger. Berlín, 1-948 pp. 